\title{
Alpha-Shapes and Flow Shapes are Homotopy Equivalent *
}

[Extended Abstract]

\author{
Tamal K. Dey \\ The Ohio State University, \\ USA \\ tamaldey@cis.ohio- \\ state.edu
}

\author{
Joachim Giesen \\ ETH Zürich, Switzerland \\ giesen@inf.ethz.ch
}

\author{
Matthias John \\ ETH Zürich, Switzerland \\ john@inf.ethz.ch
}

\begin{abstract}
In this paper we establish a topological similarity between two apparently different shape constructors from a set of points. Shape constructors are geometric structures that transform finite point sets into continuous shapes. Due to their immense practical importance in geometric modeling various shape constructors have been proposed recently. Understanding the relations among them often leads to new insights that are potentially helpful in applications. Here we discover a topological equivalence among two such geometric structures, namely $\alpha$-shapes and flow shapes. Both shapes found applications in surface reconstruction and molecular modeling
\end{abstract}

\section{Categories and Subject Descriptors}

F.2.2 [Nonnumerical Algorithms and Problems]: Geometrical problems and computations; I.3.5 [Computational Geometry and Object Modeling]: Curve, surface, solid, and object representations

\section{General Terms}

Theory, Algorithms

\section{INTRODUCTION}

Sample based shape modeling has gained popularity in recent years because of its wide applicability in science and engineering $[1,2,3,4,7]$. For example, it is almost routine to obtain sample points from the boundary of an object with recent scanning devices. Shape modeling reverses

\begin{abstract}
* Partly supported by the IST Programme of the EU and the Swiss Federal Office for Education and Science as a Shared-cost RTD (FET Open) Project under Contract No IST-2000-26473 (ECG - Effective Computational Geometry for Curves and Surfaces). Also supported by NSF under the subcontract of the grant DMS-0138456.
\end{abstract}

Permission to make digital or hard copies of all or part of this work for personal or classroom use is granted without fee provided that copies are not made or distributed for profit or commercial advantage and that copies bear this notice and the full citation on the first page. To copy otherwise, to republish, to post on servers or to redistribute to lists, requires prior specific permission and/or a fee.

STOC'03, June 9-11, 2003, San Diego, California, USA

Copyright 2003 ACM 1-58113-674-9/03/0006 ...\$5.00. this process of discretization, that is, it creates a continuous shape out of these discrete points. The $\alpha$-shapes originally proposed by Edelsbrunner et al. [8] and later extended by Edelsbrunner and Mücke [10] provide an efficient means for creating shapes out of point sets. Recently, flow shapes discovered by Giesen and John [11] and also by Edelsbrunner [6] provide another means of creating shapes out of a point set. In fact both $\alpha$-shapes and flow shapes can be used to define a hierarchy of shapes from a set of points. This allows multi scale modeling which turns out to be useful in detecting features at different length scales such as detecting pockets in macromolecules [7].

$\alpha$-shapes put a ball of radius $\sqrt{\alpha}$ around each point and construct a simplicial complex that respects the intersections among these balls. The underlying space of this simplicial complex is defined as the $\alpha$-shape. As $\alpha$ changes, new simplices are added or deleted as intersections among the balls appear or disappear. This means a hierarchy of shapes can be defined using $\alpha$ as a scale parameter. Flow shapes, on the other hand, are defined as a cell decomposition of the embedding space of the sample points. The decomposition is based on the gradient flow of a distance function. The cells of this decomposition can be ordered by some distance values giving a hierarchy of shapes.

While both $\alpha$-shapes and flow shapes define a hierarchy of shapes using some scale parameter, they are quite different geometrically. We illustrate this fact later using an example data set. Naturally a relevant question is raised whether the two hierarchy have any kind of similarity. We show that indeed the two hierarchies have a certain topological similarity, namely they are homotopy equivalent. Specifically, both $\alpha$-shapes and flow shapes change their topology only at discrete critical levels in the hierarchy. These critical levels turn out to be the same in both hierarchies. We show that at any critical level the two shapes are homotopy equivalent. The homotopy equivalence between two shapes holds even between two consecutive critical levels. However, there is a striking difference between $\alpha$-shapes and flow shapes in these intervals between critical levels. Many $\alpha$-shapes that are different as sets but have the same homotopy type may appear in these intervals while different flow shapes only appear at critical levels. Our result shows that, even in the presence of this difference one does not miss any topological change captured by the $\alpha$-shape hierarchy when taking the flow shape hierarchy instead. This sparseness of the flow shape hierarchy should be beneficial for some applications. Furthermore the definition of flow shapes has a strong Morse theoretic 
flavor which might allow the use of Morse theoretic concepts that are not directly applicable to the $\alpha$-shapes. Also, many applications of $\alpha$-shapes may benefit from taking the advantages of flow shapes which sometimes seem to capture geometry better as we will demonstrate later in Figure 11.

\section{COMPLEXES}

The $\alpha$-shape and the flow shape are the underlying spaces of cell complexes called the $\alpha$-complex and the flow complex, respectively. These cell complexes are derived more or less directly from the Voronoi- and Delaunay complexes of a finite set of points in $\mathbb{R}^{3}$. In this section we summarize the definitions of Voronoi- and Delaunay complexes and give the definition of an $\alpha$-complex and an $\alpha$-shape associated with a finite set of points. The definition of an $\alpha$-complex is very similar to the definition of the Delaunay complex. This is not the case for the flow complex. Thus we introduce the flow complex in a separate section.

Voronoi complex. Let $P$ be a finite set of points in $\mathbb{R}^{3}$. The Voronoi cell of $p \in P$ is given as

$$
\left.V_{p}=\left\{x \in \mathbb{R}^{3}: \forall q \in P-\{p\},\|x-p\| \leq\|x-q\|\right)\right\} .
$$

The sets $V_{p}$ are convex polyhedra or empty since the set of points that have the same distance from two points in $P$ forms a hyperplane. Closed facets shared by two Voronoi cells are called Voronoi facets, closed edges shared by three or more Voronoi cells are called Voronoi edges and the points shared by four or more Voronoi cells are called Voronoi vertices. The term Voronoi object denotes either a Voronoi cell, facet, edge or vertex. The Voronoi complex $V(P)$ of $P$ is the collection of all Voronoi objects. It defines a cell decomposition of $\mathbb{R}^{3}$.

Delaunay complex. The Delaunay complex of a set of points $P$ is dual to the Voronoi complex of $P$. The convex hull of four or more points in $P$ defines a Delaunay cell if the intersection of the corresponding Voronoi cells is not empty and there exists no superset of points in $P$ with the same property. Analogously, the convex hull of three or two points defines a Delaunay face or Delaunay edge, respectively, if the intersection of their corresponding Voronoi cells is not empty. Every point in $P$ is called Delaunay vertex. The term Delaunay object denote either a Delaunay cell, face, edge or vertex. The Delaunay complex $D(P)$ defines a decomposition of the convex hull of all points in $P$. This decomposition is a triangulation if the points are in general position.

We always refer to the interior and to the boundary of Voronoi-/Delaunay objects with respect to their dimension, e.g. the interior of a Delaunay edge contains all points in this edge besides the endpoints and the interior of a vertex and its boundary are the vertex itself. Furthermore, we always assume general position unless stated differently.

Union of balls. Let $P$ be a finite set of points in $\mathbb{R}^{3}$. The union of balls centered at the points in $P$ with radius $\sqrt{\alpha}$ for $\alpha \geq 0$ is denoted by $B^{\alpha}(P)$, i.e.

$$
B^{\alpha}(P)=\left\{x \in \mathbb{R}^{3}: \exists p \in P \text { such that }\|p-x\|^{2} \leq \alpha\right\} .
$$

$\alpha$-complex. Given a finite set of points $P \in \mathbb{R}^{3}$ and $\alpha \geq$ 0 , the $\alpha$-complex of $P$ is the dual complex of the Voronoi diagram of $P$ restricted to the union of balls $B^{\alpha}(P)$. The restricted Voronoi cell of $p \in P$ is given as

$$
V_{p}^{\alpha}=V_{p} \cap B^{\alpha}(P) .
$$

The restricted Voronoi cells are used to define the $\alpha$-complex $K^{\alpha}(P)$ analogously to the Delaunay complex. The convex hull of four or more points in $P$ defines a face in the $\alpha$ complex if the intersection of the corresponding restricted Voronoi cells is not empty and there exists no superset of points in $P$ with the same property. Similarly, the convex hull of $k, 1 \leq k \leq 3$ defines a facet in the $\alpha$-complex if the intersection of the corresponding restricted Voronoi cells is not empty.

By construction the $\alpha$-complex is a sub-complex of the Delaunay complex for every $\alpha \geq 0$. With increasing $\alpha$ more and more cells of the Delaunay complex appear in the $\alpha$-complex, i.e. $K^{\alpha}(P) \subseteq K^{\alpha^{\prime}}(P)$ for $\alpha \leq \alpha^{\prime}$. In fact, we can get a filtration of the Delaunay complex from the $\alpha$-complexes, i.e. a sequence $K^{\alpha_{1}}(P), \ldots, K^{\alpha_{n}}(P)$ of $\alpha$-complexes such that

$$
P=K^{\alpha_{1}=0}(P) \subset K^{\alpha_{2}}(P) \subset \ldots \subset K^{\alpha_{n}}(P)=D(P) .
$$

The $\alpha_{i}, i>1$ are typically chosen to be the critical $\alpha$-levels. For convenience we extend the definition of $\alpha$-complexes to negative values of $\alpha$ by setting $K^{\alpha}(P)=K^{0}(P)=P$ for all $\alpha<0$.

Critical $\alpha$-level. Let $P$ be a finite point set in $\mathbb{R}^{3}$. A value $\alpha \in(0, \infty)$ is called a critical $\alpha$-level if $K^{\alpha-\varepsilon}(P) \neq K^{\alpha}(P)$ for all $\varepsilon>0$.

It is important to note that the homotopy type of the $\alpha$ shape changes only at critical $\alpha$-levels, but it is easy to see that there are critical $\alpha$-levels where the homotopy type of the corresponding $\alpha$-shape does not change, see Figure 1 for an example.

The following theorem is due to Edelsbrunner [5]. It can be proven using the nerve theorem, but Edelsbrunner also gave a deformation retraction from $B^{\alpha}(P)$ to the corresponding $\alpha$-shape.

TheOREM 1. Given a finite set of points $P \subset \mathbb{R}^{3}$. For every $\alpha \geq 0$ the union of balls $B^{\alpha}(P)$ and the $\alpha$-shape corresponding to $K^{\alpha}(P)$ are homotopy equivalent.

In the subsequent sections we are going to associate with every value $\alpha \in[0, \infty)$ for a given point set $P$ another cell complex $F^{\alpha}(P)$, namely the flow complex at level $\alpha$. The main theorem of this paper then states that the underlying topological spaces of $K^{\alpha}(P)$ and $F^{\alpha}(P)$ are homotopy equivalent for every $\alpha \in[0, \infty)$.

\section{INDUCED FLOW}

The flow that we are going to study is the flow along the gradient vector field of the distance function induced by a finite set of sample points. Extra care has to be taken since this distance function is not smooth everywhere, i.e. the gradient is not defined everywhere. 

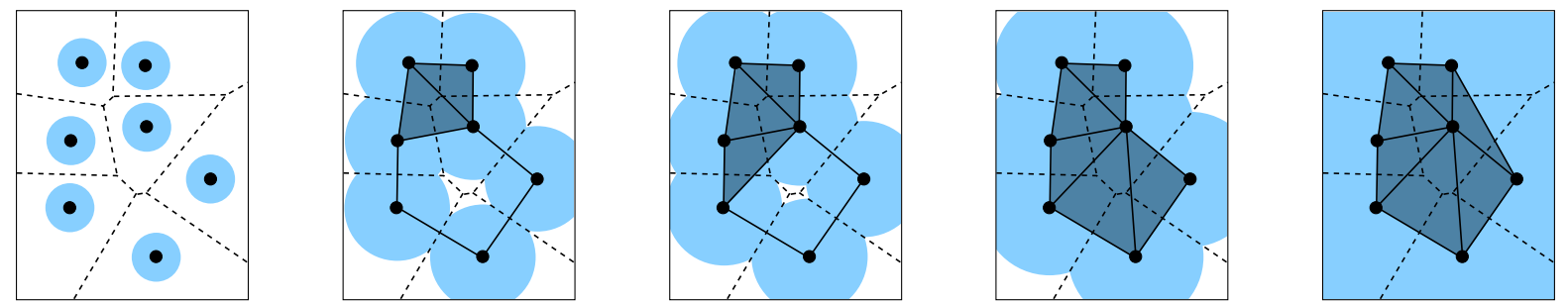

Figure 1: From the left to the right are shown $\alpha$-complexes for growing values of $\alpha$. Note that the $\alpha$-complexes shown as second from the left and in the middle have the same homotopy type though they are different complexes. The same holds for the two rightmost complexes.

Distance function. Let $P$ be a finite set of points in $\mathbb{R}^{3}$. The distance function induced by $P$ is given as

$$
h(x)=\min \left\{\|x-p\|^{2}: p \in P\right\} .
$$

The graph of the distance function $h$ is the lower envelope of a set of paraboloids centered at the points in $P$. Thus the function $h$ is continuous. It is smooth everywhere besides at points which have the same distance from two or more points, i.e. at points that lie on the boundary of a Voronoi cell.

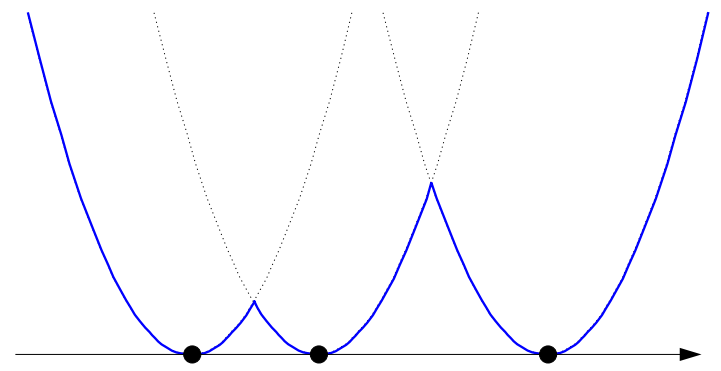

Figure 2: A one dimensional example that shows the graph (solid line) of the distance function induced by three points.

The definitions given in the critical point theory of distance functions developed in Riemannian geometry [12] boils down in our setting to the following:

Regular- and critical points. Let $P$ be a finite set of points such that Voronoi and their dual Delaunay objects intersect in their interiors if they intersect at all. Then the critical points of the distance function $h$ are the intersection points of Voronoi objects $V$ and their dual Delaunay object $\sigma$. The index of a critical point is the dimension of $\sigma$. Non critical points are called regular.

Note that the intuition about critical points that they are local extrema and saddle points is still valid in this more general setting.

In the following we always assume that Voronoi and their dual Delaunay objects intersect in their interiors if they in- tersect at all. Other intersections are degenerate in the sense that they are stable under small perturbations of the point set.

Critical level. Let $P$ be a finite set of points. A value $\alpha \in[0, \infty)$ is called a critical level of the distance function $h$ associated with $P$ if $h^{-1}(\alpha)$ contains a critical point of $h$.

TheOREM 2. Let $P$ be a finite set of points in $\mathbb{R}^{3}$ and let $h$ be the corresponding distance function. If the interval $\left[\alpha, \alpha^{\prime}\right] \subset[0, \infty)$ does not contain any critical level then $h^{-1}([0, \alpha])$ is homeomorphic to $h^{-1}\left(\left[0, \alpha^{\prime}\right]\right)$ and $h^{-1}\left(\left[0, \alpha^{\prime}\right]\right)$ deformation retracts to $h^{-1}([0, \alpha])$.

Proof. This theorem is the specialization of the main theorem of the critical point theory of distance functions [12] to the distance function $h$.

Note that the union of balls $B^{\alpha}(P)$ is just the set $h^{-1}(\alpha)$. Thus we get from Theorem 2 that the homotopy type of the union of balls $B^{\alpha}(P)$ changes only at the critical levels of the distance function $h$. Together with Theorem 1 this implies that the homotopy type of the $\alpha$-shapes only changes at the critical levels of $h$. Hence the critical levels of $h$ are all critical $\alpha$-levels. Note that the converse is not true, i.e. not every critical $\alpha$-level is a critical level of $h$, see Figure 1 for a counterexample.

The following definition turns out to be very helpful in the subsequent discussion. It allows us to characterize the direction of steepest ascent of the distance function $h$ at every point $x \in \mathbb{R}^{3}$.

Driver. Let $x \in \mathbb{R}^{3}$ be any point. Let $V$ be the lowest dimensional Voronoi object in the Voronoi complex of $P$ that contains $x$ and let $\sigma$ be the dual Delaunay object of $V$. The driver $d(x)$ of $x$ is the point on $\sigma$ closest to $x$.

It can be shown at every regular point $x \in \mathbb{R}^{3}$ the direction of steepest ascent of the distance function $h$ is given by the vector

$$
v(x)=\frac{x-d(x)}{\|x-d(x)\|} .
$$

We want to study how the points in $\mathbb{R}^{3}$ move if they always follow the direction of steepest ascent. The curve that 
a point $x \in \mathbb{R}^{3}$ follows in this motion is called the orbit of $x$. For smooth distance functions the computation of a single orbit results in the solution of an ordinary differential equation. Since the distance function $h$ is not smooth everywhere, we cannot apply the theory of ordinary differential equations here. Nevertheless individual orbits can also be computed for $h$. They can be derived from the flow induced by the point set $P$. The induced flow is a solution $\phi:[0, \infty) \times \mathbb{R}^{3} \rightarrow \mathbb{R}^{3}$ of the following equation:

$$
\lim _{t \downarrow t_{0}} \frac{\phi(t, x)-\phi\left(t_{0}, x\right)}{t-t_{0}}=v\left(\phi\left(t_{0}, x\right)\right)
$$

One can easily check that the following definition satisfies the equation above. Note that the definition is inherently algorithmic.

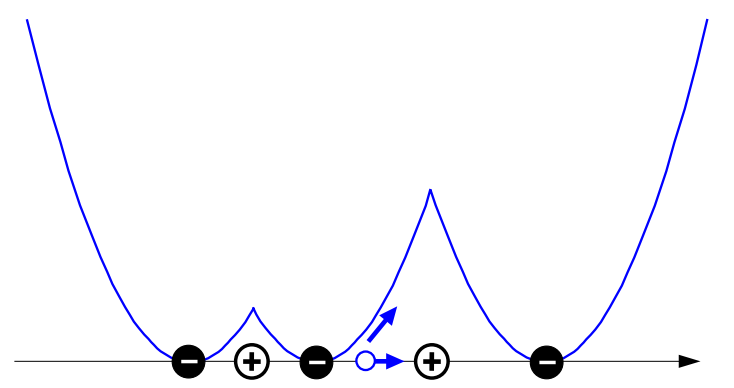

Figure 3: The critical points of the distance function from Figure 2 and the direction of steepest ascent of the distance function at one point. Note that in one dimension the only critical points of the distance function are local minima $\ominus$ and local maxima $\oplus$.

Induced flow. The flow $\phi$ induced by a finite point set $P$ is given as follows: For all critical points $x$ of the height function associated with $P$ we set:

$$
\phi(t, x)=x, t \in[0, \infty)
$$

Otherwise let $d(x)$ be the driver of $x$ and $R$ be the ray originating at $x$ and shooting in the direction $v(x)=x-$ $d(x) /\|x-d(x)\|$. Let $z$ be the first point on $R$ whose driver is different from $d(x)$. Note that such a $z$ need not exist in $\mathbb{R}^{3}$ if $x$ is contained in an unbounded Voronoi object. In this case let $z$ be the point at infinity in the direction of $R$. We set:

$$
\phi(t, x)=x+t \cdot v(x), t \in[0,\|z-x\|)
$$

For $t \geq\|z-x\|$ the flow is given as follows:

$$
\begin{aligned}
\phi(t, x) & =\phi(t-\|z-x\|+\|z-x\|, x) \\
& =\phi(t-\|z-x\|, \phi(\|z-x\|, x))
\end{aligned}
$$

It is shown in [11] that the function $\phi$ is well defined.

Orbits and fixpoints. Given $x \in \mathbb{R}^{3}$ and an induced flow $\phi$, the curve

$$
\phi_{x}:[0, \infty) \rightarrow \mathbb{R}^{3}, t \mapsto \phi(t, x)
$$

is called the orbit of $x$. A point $x \in \mathbb{R}^{3}$ is called a fixpoint of $\phi$ if $\phi_{x}(t)=x$ for all $t \geq 0$.

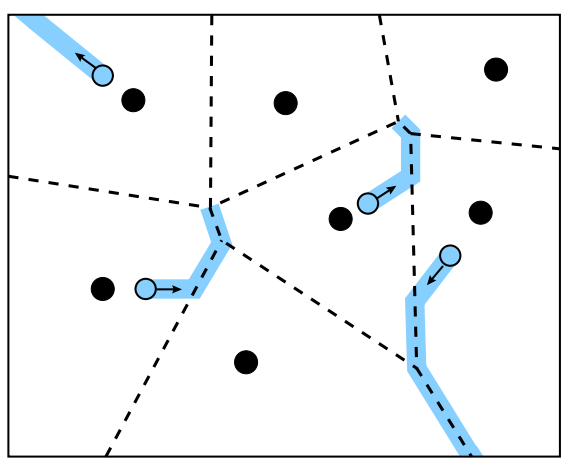

Figure 4: An example that shows four orbits of a flow induced by seven points in the plane. The plane is clipped with a rectangle. The Voronoi complex of the point set is also shown (dashed lines).

OBSERVATION 1. The fixpoints of $\phi$ are the critical points of the distance function $h$.

Because of this observation we refer to a fixpoint of $\phi$ as a minimum, saddle or maximum if the corresponding critical point of the distance function is a minimum, saddle point or maximum, respectively.

\section{FLOW COMPLEX}

We are going to group all points together that flow into the same fixpoint of the flow. These inflow regions of critical points are essentially the cells of the flow complex that we are also going to define in this section.

Stable manifolds. Given an induced flow $\phi$, the stable manifold $S(x)$ of a fixpoint $x \in \mathbb{R}^{3}$ is the set of all points that flow into $x$, i.e.

$$
S(x)=\left\{y \in \mathbb{R}^{3}: \lim _{t \rightarrow \infty} \phi_{y}(t)=x\right\} .
$$

Instead of directly working with stable manifolds of critical points we introduce a smoothed version which has nicer properties. Smoothing means for all practical purposes taking the closure of the stable manifold. We will later comment on why the following definition is more complicated.

Smoothed stable manifolds. Let $x$ be a fixpoint of index $i$ of an induced flow. That is, the corresponding critical point of the distance function $h$ has index $i$. Let $S$ be the set of points in $S(x)$ that have a neighborhood in $S(x)$ that is homeomorphic to an open subset of $\mathbb{R}^{3}, d=i+1, \ldots, 3$. Let $S^{\prime}$ be the boundary of $S(x)-S$ in $\mathbb{R}^{3}$. The smoothed stable manifold of $x$ is the set $S^{*}(x)=(S(x)-S) \cup S^{\prime}$.

Flow complex. Given the flow $\phi$ induced by a finite point set $P \subset \mathbb{R}^{3}$ and $\alpha \geq 0$, the flow complex $F^{\alpha}(P)$ is defined as the collection of all stable manifolds of critical points $x$ with $h(x) \leq \alpha$. For convenience we also extend this definition to negative values of $\alpha$ be setting $F^{\alpha}(P)=F^{0}(P)=P$ for $\alpha<0$. We refer to the underlying topological space of 
$F^{\alpha}(P)$ as flow shape.

The definition of flow complexes corresponding to a finite point set $P \subset \mathbb{R}^{3}$ implies that

$$
F^{\alpha}(P) \subseteq F^{\alpha^{\prime}}(P) \text { for } \alpha \leq \alpha^{\prime} .
$$

By construction the values $\alpha \geq 0$ such that $F^{\alpha-\varepsilon}(P) \neq$ $F^{\alpha}(P)$ for all $\varepsilon>0$ are exactly the critical levels of the distance function $h$. Thus we have the following observation.

Observation 2. Let $P \subset \mathbb{R}^{3}$ finite set of points. The homotopy type of the flow shape changes only at the critical levels of the distance function $h$ induced by $P$.

In the following we will show that the flow complex is actually a cell complex for every $\alpha \in[0, \infty)$. An induced flow in $\mathbb{R}^{3}$ has four different types of fixpoints, local minima, saddle points of index 1 , saddle points of index 2 and local maxima. In the following we are going to characterize the smoothed stable manifolds of the four different types of fixpoints.

OBSERVATION 3. The smoothed stable manifold of a local minimum $m$ contains just the point $m$ itself since no other point flows into $m$.

It turns out that the stable manifold of an index 1 saddle point is always a Gabriel edge and vice versa.

Gabriel graph. The Gabriel graph of a finite set of points $P$ in $\mathbb{R}^{3}$ is given as follows: Its vertices are the points in $P$ and its edges are given by Delaunay edges that intersect their dual Voronoi facet. The edges of the Gabriel graph are called Gabriel edges. The Gabriel graph is always connected, because it contains the Euclidean minimum spanning tree of $P$.

LEMma 1. Let $s$ be an index 1 saddle of $\phi$. The smoothed stable manifold $S^{*}(s)$ of $s$ is a Gabriel edge and every Gabriel edge is the smoothed stable manifold of some index 1 saddle.

The smoothed stable manifolds of index 2 saddle points have a more complicated structure. Note that, in general, they are not composed of Delaunay triangles.

Lemma 2. Let $s$ be an index 2 saddle of $\phi$. If the stable manifold $S(s)$ of $s$ does not contain a Voronoi vertex then $S^{*}(s)$ is a piecewise linear surface with boundary. The boundary of the surface is made up of Gabriel edges.

Proof. A constructive proof can be found in [11]. Later we need some ideas from the constructions in this proof to prove the main result of this paper, i.e. the homotopy equivalence of $\alpha$-shapes and flow shapes.

Note that we do not claim that the surface is homeomorphic to a disk. In fact, the surface need not be simply connected, i.e. it can have holes. Furthermore, the lemma does no longer hold if the stable manifold $S(s)$ contains a Voronoi vertex. In that case $S(s)$ can have three dimensional components which forced us to give the quite complicated definition of smoothed stable manifolds. This definition takes care of the three dimensional parts. But in the following we want to assume that none of the stable manifolds of index 2 saddle points contains a Voronoi vertex.

Saddle complex. Given a finite set of points in $\mathbb{R}^{3}$, we call the simplicial complex built by the smoothed stable manifolds of all saddle points, i.e. the Gabriel edges and the surfaces from Lemma 2 the saddle complex of the point set.

It remains to characterize the stable manifolds of the maxima. The proof of the following theorem appeared in [11].

THEOREM 3. The smoothed stable manifolds of the maxima of $\phi$ are exactly the closures of the bounded regions of the saddle complex provided no stable manifold of an index 2 saddle contains a Voronoi vertex.

Especially, this theorem states that the boundary of the smoothed stable manifold of a maximum is made up of the smoothed stable manifolds of index 2 saddle points. That is, under our non-degeneracy assumptions the smoothed stable manifolds of critical points of different index have a nice recursive structure. The boundary of a smoothed stable manifold of an index $d$ critical point, $1 \leq d \leq 3$, is made up of the smoothed stable manifolds of index $d-1$ critical points.

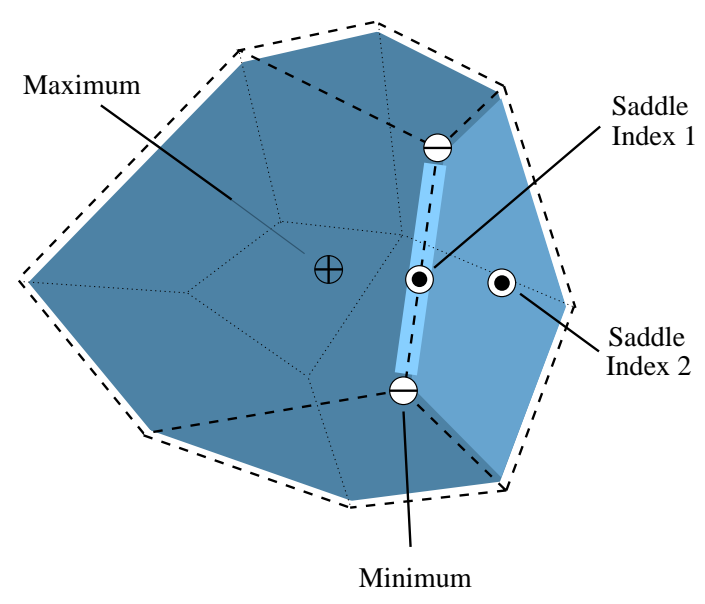

Figure 5: This figure illustrates the recursive structure of the smoothed stable manifolds of critical points of different indices. The shaded region shows the interior of a smoothed stable manifold of a maximum $\oplus$ whose boundary is made up of smoothed stable manifolds of index 2 saddle points. One such index 2 saddle point $\odot$ is shown along with the interior of its smoothed stable manifold. Also an index 1 saddle point $\odot$ in the boundary of the latter stable manifold is shown. The boundary of the smoothed stable manifold of this saddle points consists of two minima $\ominus$.

OBSERVATION 4. Let $h$ be the distance function associated with a finite set of points $P \subset \mathbb{R}^{3}$. For every critical point $y$ of $h$ in the boundary of the smoothed stable manifold of a critical point $x$ it is $h(y)<h(x)$. Thus every cell in the flow complex appears only at larger values of $\alpha$ than the cells in its boundary. 


\section{HOMOTOPY EQUIVALENCE}

In this section we are going to show that the underlying topological spaces of $K^{\alpha}(P)$ and $F^{\alpha}(P)$ are homotopy equivalent for every finite point set $P \subset \mathbb{R}^{3}$ and every $\alpha \geq 0$.

THEOREM 4. Given a finite set of points $P \subset \mathbb{R}^{3}$. For every $\alpha \geq 0$ the topological spaces underlying $K^{\alpha}(P)$ and $F^{\alpha}(P)$ are homotopy equivalent.

Proof. From Theorem 1 we know that $B^{\alpha}(P)$ and $K^{\alpha}(P)$ are homotopy equivalent for every $\alpha \geq 0$. Thus it is enough to show that $B^{\alpha}(P)$ and $F^{\alpha}(P)$ are homotopy equivalent for every $\alpha \geq 0$. We are going to prove this by induction over the critical levels of the distance function $h$ corresponding to $P$. For $\alpha=0$ we have

$$
B^{0}(P)=F^{0}(P)=P .
$$

Hence we have the stronger statement that for $\alpha=0$ the underlying topological space of $F^{0}(P)$ is not only homotopy equivalent to $B^{0}(P)$ but the two spaces coincide. In the following we will use for convenience the notation $F^{\alpha}(P)$ for the complex as well as for its underlying topological space. From Theorem 2 and Observation 2 we know that the homotopy type of $B^{\alpha}(P)$ and $F^{\alpha}(P)$ can only change at the critical levels of the distance function $h$. That is, we have to show that when $\alpha$ passes through a critical level the homotopy types of $B^{\alpha}(P)$ and $F^{\alpha}(P)$ change in the same way.

Let $0<\alpha_{1}, \ldots, \alpha_{n}$ be the critical levels of the distance function $h$. We now assume that $B^{\alpha}(P)$ and $F^{\alpha}(P)$ are homotopy equivalent for all $\alpha \leq \alpha_{i-1}+\varepsilon$, where $\varepsilon>0$ is chosen such that the interval $\left[\alpha_{i-1}-\varepsilon, \alpha_{i-1}+\varepsilon\right]$ contains no critical level besides $\alpha_{i-1}$ and the interval $\left[\alpha_{i}-\varepsilon, \alpha_{i}+\varepsilon\right]$ contains no critical level besides $\alpha_{i}$. We want to show inductively that $B^{\alpha}(P)$ and $F^{\alpha}(P)$ also have to be homotopy equivalent for all $0 \leq \alpha \leq \alpha_{i}+\varepsilon$.

Let $x \in h^{-1}\left(\alpha_{i}\right)$ be a critical point of $h$. We can assume without loss of generality that $x$ is the only critical point in $h^{-1}\left(\alpha_{i}\right)$, because all operations that we are going to perform in the following can be localized around each critical point in $h^{-1}\left(\alpha_{i}\right)$ by using a suited partition of unity. Since $\alpha_{i}>0$ the point $x$ cannot be a minimum, i.e. it is either a saddle point or a local maximum. We distinguish three cases. Either $x$ is an index 1 saddle point, an index 2 saddle point or a maximum.

First CASE. Assume that $x$ is an index 1 saddle point. Our characterization of critical points states that $x$ has to be contained in a Delaunay edge $E$ that intersects its dual Voronoi facet, i.e. $x$ is contained in a Gabriel edge. For $\varepsilon>0$ sufficiently small the Gabriel edge $E$ is completely covered by the union of balls $B^{\alpha_{i}-\varepsilon}(P)$ besides a small open line segment contained in $E$ that contains $x$. Let $E^{\prime}$ be the closure of this line segment and let $v$ and $w$ be the two endpoints of $E^{\prime}$.

We have $B^{\alpha_{i}-\varepsilon}(P) \cap e^{\prime}=\{v, w\}$. Thus the map

$$
\varphi: \operatorname{boundary}\left(e^{\prime}\right)=\{v, w\} \rightarrow B^{\alpha_{i}-\varepsilon}(P)
$$

that maps $v$ and $w$ to itself can be used to glue $E^{\prime}$ into $B^{\alpha_{i}-\varepsilon}(P)$. The topological space that results from this gluing operation is denoted by $B^{\alpha_{i}-\varepsilon}(P) \cup_{\varphi} E^{\prime}$. Siersma proves in [14] that $B^{\alpha_{i}+\varepsilon}(P)$ is homotopy equivalent to $B^{\alpha_{i}-\varepsilon}(P) \cup_{\varphi}$

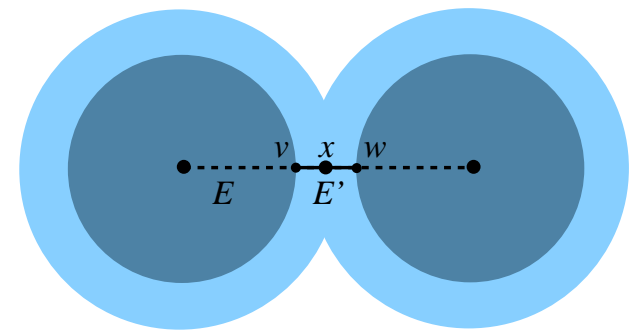

Figure 6: Shown in two dimensions is the Gabriel edge $E$ that contains the index 1 saddle point $x$. Also shown are two balls of the union of balls $B^{\alpha_{i}-\varepsilon}(P)$ (dark shaded) and $B^{\alpha_{i}+\varepsilon}(P)$ (light shaded) and the line segment $E^{\prime} \subset E$ with its endpoints $v$ and $w$.

$E^{\prime}$ by giving an explicit deformation retraction of $B^{\alpha_{i}+\varepsilon}(P)$ to $B^{\alpha_{i}-\varepsilon}(P) \cup_{\varphi} E^{\prime}$. Actually, Siersma provides a prove only for the two dimensional case but its generalization to three dimensions is straightforward. From Theorem 2 we know that $B^{\alpha_{i}-\varepsilon}(P)=h^{-1}\left(\left[0, \alpha_{i}-\varepsilon\right]\right)$ is homotopy equivalent to $B^{\alpha_{i-1}+\varepsilon}(P)=h^{-1}\left(\left[0, \alpha_{i-1}+\varepsilon\right]\right)$. The latter union of balls is by our inductive assumption homotopy equivalent to $F^{\alpha_{i-1}+\varepsilon}(P)$ which in turn is the same as $F^{\alpha_{i-1}}(P)$ since $\alpha_{i-1}$ is the only critical level contained in the interval $\left[\alpha_{i-1}, \alpha_{i-1}+\varepsilon\right]$. By the definition of $E^{\prime}$ we have $E^{\prime} \subset F^{\alpha_{i}}$. That is, we can look at $F^{\alpha_{i}}(P)$ as the topological space we get by gluing $E^{\prime}$ into closure $\left(F^{\alpha_{i}}(P)-E^{\prime}\right)$. The gluing is done by mapping $v$ and $w$ in $E^{\prime}$ to $v$ and $w$ in $F^{\alpha_{i}-\varepsilon}(P)$. There is a straightforward deformation retraction of $\operatorname{closure}\left(F^{\alpha_{i}}(P)-E^{\prime}\right)$ to $F^{\alpha_{i-1}}$ by just pulling back the endpoints $v$ and $w$ of $E^{\prime}$ to the corresponding endpoints of the Gabriel edge $E$. Thus we find that $F^{\alpha_{i}}(P)-E^{\prime}$ and $F^{\alpha_{i-1}}(P)$ are homotopy equivalent.This provides us with the following sequence of homotopy equivalences:

$$
\begin{aligned}
F^{\alpha_{i}}(P)-E^{\prime} & \simeq F^{\alpha_{i-1}}(P) \\
& =F^{\alpha_{i-1}+\varepsilon}(P) \\
& \simeq B^{\alpha_{i-1}+\varepsilon}(P) \\
& =h^{-1}\left(\left[0, \alpha_{i-1}+\varepsilon\right]\right) \\
& \simeq h^{-1}\left(\left[0, \alpha_{i}-\varepsilon\right]\right) \\
& =B^{\alpha_{i}-\varepsilon}(P)
\end{aligned}
$$

By gluing the same segment $E^{\prime}$ at the same points $v$ and $w$ into the two homotopy equivalent spaces $F^{\alpha_{i}}(P)-E^{\prime}$ and $B^{\alpha_{i}-\varepsilon}(P)$ we get a homotopy equivalence,

$$
F^{\alpha_{i}}(P) \simeq B^{\alpha_{i}-\varepsilon}(P) \cup_{\varphi} E^{\prime} .
$$

That is, altogether we have the following sequence of homotopy equivalences:

$$
\begin{aligned}
B^{\alpha_{i}+\varepsilon}(P) & \simeq B^{\alpha_{i}-\varepsilon}(P) \cup_{\varphi} E^{\prime} \\
& \simeq F^{\alpha_{i}}(P) \\
& =F^{\alpha_{i}+\varepsilon}(P)
\end{aligned}
$$

Hence the the union of balls $B^{\alpha_{i}+\varepsilon}(P)$ and the flow shape $F^{\alpha_{i}+\varepsilon}(P)$ have the same homotopy type at level $\alpha_{i}+\varepsilon$. Since we know that the flow shape and the union of balls can change their homotopy type in the interval $\left[\alpha_{i}-\varepsilon, \alpha_{i}+\varepsilon\right]$ only at the critical level $\alpha_{i}$ we have that $B^{\alpha}(P)$ and $F^{\alpha}(P)$ 
are homotopy equivalent for all $0 \leq \alpha \leq \alpha_{i}+\varepsilon$.

We want to use the same idea of proof also for the case that the critical point $x$ is either an index 2 saddle point or a local maximum. It turns out that the proof can be copied almost line by line for the remaining two cases besides the part that establishes the homotopy equivalence

$$
F^{\alpha_{i}}(P)-E^{\prime} \simeq F^{\alpha_{i-1}}(P),
$$

where $E^{\prime}$ is replaced either by a small surface patch if $x$ is an index 2 saddle or by a small volume element if $x$ is a local maximum. The proof of this homotopy equivalence was trivial for the case that $x$ is an index 1 saddle point. It turns out that we have to work harder to prove the analogous statements in the remaining cases.

SECOND CASE. Now assume that $x$ is an index 2 saddle point. Our characterization of critical points states that $x$ has to be contained in a Delaunay facet $F$ that is intersected by its dual Voronoi edge. For $\varepsilon>0$ sufficiently small the Delaunay facet $F$ is completely covered by the union of balls $B^{\alpha_{i}-\varepsilon}$ besides a small open subset contained in the interior of $F$ that contains $x$. Let $F^{\prime}$ be the closure of this subset in $F$.

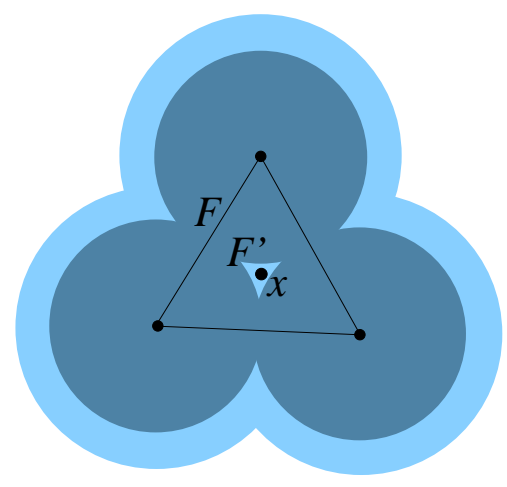

Figure 7: Shown in two dimensions is the Delaunay triangle $F$ that contains the index 2 saddle point $x$. Also shown are three balls of the union of balls $B^{\alpha_{i}-\varepsilon}(P)$ (dark shaded) and $B^{\alpha_{i}+\varepsilon}(P)$ (light shaded) and the surface patch $F^{\prime} \subset F$ (also light shaded).

The proof of Lemma 2, see [11] tells us that we have $F^{\prime} \subset$ $F^{\alpha_{i}}(P)$ if we choose $\varepsilon$ sufficiently small. The boundary of $F^{\prime}$ is by definition contained in $\operatorname{closure}\left(F^{\alpha_{i}}(P)-F^{\prime}\right)$ but it is also contained in $B^{\alpha_{i}-\varepsilon}(P)$. That is we can glue $F^{\prime}$ along its boundary into both spaces. Using again an explicit deformation retraction similar to one described in [14] we can establish the homotopy equivalence

$$
B^{\alpha_{i}+\varepsilon}(P) \simeq B^{\alpha_{i}-\varepsilon}(P) \cup_{\varphi} F^{\prime},
$$

where $\varphi$ maps the boundary of $F^{\prime}$ onto its copy contained in $B^{\alpha_{i}-\varepsilon}(P)$. If we show that

$$
F^{\alpha_{i}}(P)-F^{\prime} \simeq F^{\alpha_{i-1}}(P),
$$

then we are done, because we can apply a similar proof as in the first case. Note that the homotopy equivalence is not obvious since the smoothed stable manifold $S^{*}(x)$ of $x$ need not be a topological disc. We are now going to prove the homotopy equivalence by giving an explicit deformation retraction. This deformation retraction will be a little bit more complicated than necessary here. The benefit of complicating things is that we can apply the strategy used here also in the case that $x$ is a local maximum.

To construct a deformation retraction of $F^{\alpha_{i}}(P)-F^{\prime}$ to $F^{\alpha_{i-1}}(P)$ we shortly recall how the smoothed stable manifold $S^{*}(x)$ of the index 2 saddle $x$ can be computed. The details can be found in the proof of Lemma 2, see [11]. We first want to identify all points in $S^{*}(x)$ that flow into $x$ on straight line segments - more specific we are interested in all these line segments and denote the set of all these line segments as $L$. For every Voronoi cell incident to $x$ the line segment connecting the dual Delaunay vertex of this Voronoi cell with $x$ is a line segment in $L$. The remaining line segments in $L$ pass through the Voronoi facets incident to $x$. For every of these Voronoi facets we get exactly one line segment in $L$ by first connecting the unique driver $d$ of the facet with $x$ and then determining the second intersection point besides $x$ of this line segment with the boundary of the Voronoi facet. In the proof of Lemma 2, see [11], this intersection point is denoted as $s^{\prime}$. For every line segment in $l \in L$ we consider a small cone with apex $y$ around $l$. Here $y$ denotes the second endpoint of the line segment besides $x$. The cones are chosen small enough such that they do not intersect each other. Every point in the interior of the intersection of such a cone with $S^{*}(x)$ can be pulled back to $y$ on a straight line. This gives us a deformation retraction of $F^{\alpha_{i}}(P)-F^{\prime}$ to $F^{\alpha_{i}}(P)-F^{\prime}$ with the interior of the intersection the cones with $S^{*}(x)$ removed. We denote the latter set as $F_{1}$. Figure 8 illustrates the deformation retraction of $F^{\alpha_{i}}(P)-F^{\prime}$ to $F_{1}$.
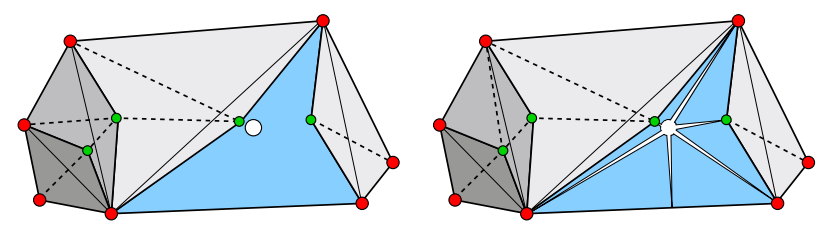

Figure 8: A schematic illustration of the deformation retraction of $F^{\alpha_{i}}(P)-F^{\prime}$ to $F_{1}$. The red points denote Delaunay vertices and the green points denote points $s^{\prime}$. We show $F^{\alpha_{i}}(P)-F^{\prime}$ on the left and $F_{1}$ on the right.

For every line segment in $L$ whose second endpoint $y$ is not a Delaunay vertex we consider two triangles $x y z$ and $x y z^{\prime}$. Here $z$ and $z^{\prime}$ are the two Delaunay vertices such that all interior points of the line segments that connect $y$ with $z$ and $z^{\prime}$, respectively, flow into $y$. The intersections of $F_{1}$ the triangles $x y z$ and $x y z^{\prime}$ can be retracted to the line segments $y z$ and $y z^{\prime}$ in a straightforward manner. We denote the set that we get after retracting all such triangles as $F_{2}$. Figure 9 (on the left) illustrates such a set $F_{2}$.

We continue the construction of the deformation retraction of $F^{\alpha_{i}}(P)-F^{\prime}$ to $F^{\alpha_{i-1}}(P)$ iteratively at every apex of the cones that is not a critical point. If such an apex is a critical point then it is already contained in the boundary of $S^{*}(x)$. Around every apex $y$ that is not critical we place a small 

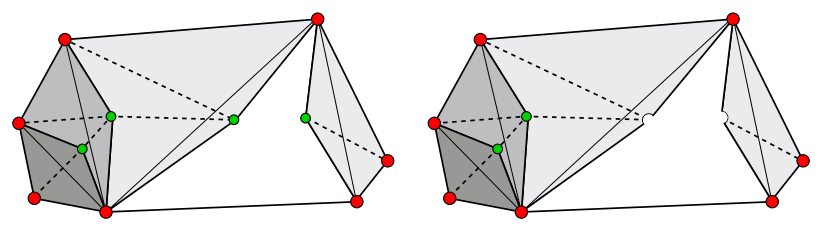

Figure 9: A schematic illustration of $F_{2}$ on the left and $F_{3}$ on the right.

ball and retract the intersection of $F_{2}$ with this ball to the intersection of $F_{2}$ the boundary of this ball. We denote the set that we get from this deformation retraction as $F_{3}$. See Figure 9 (on the right) for an illustration of this straight forward deformation retraction of $F_{2}$ to $F_{3}$. We can now proceed iteratively by processing $y$ until there is no further point to process. Figure 10 shows two more steps in such an iteration.
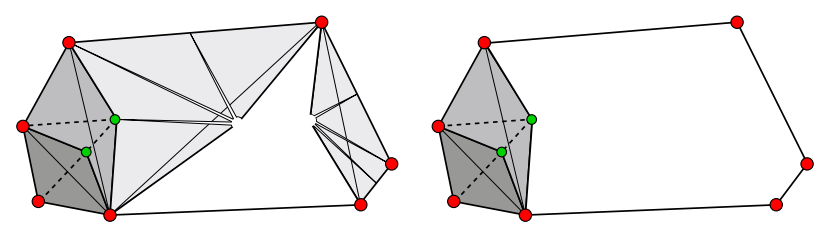

Figure 10: Two further steps in the deformation retraction of $F^{\alpha_{i}}(P)-F^{\prime}$ to $F^{\alpha_{i-1}}(P)$ starting at $F_{3}$.

From this procedure we get a sequence of sets

$$
F^{\alpha_{i}}(P)-F^{\prime}=F_{0}, F_{1}, \ldots, F_{m}=F^{\alpha_{i-1}}(P)
$$

where each $F_{i+1} \subset F_{i}$ is a deformation retract of $F_{i}$. Thus the whole sequence provides with us with the homotopy equivalence of $F^{\alpha_{i}}(P)-F^{\prime}$ and $F^{\alpha_{i-1}}(P)$. That finishes the case that $x$ is an index 2 saddle point.

ThIRD CASE. Finally assume that $x$ is a local maximum. Our characterization of critical points states that $x$ is a Voronoi vertex contained in its dual Delaunay cell $T$. For $\varepsilon>0$ sufficiently small the Delaunay cell $T$ is completely covered by the union of balls $B^{\alpha_{i}-\varepsilon}$ besides a small open subset contained in the interior of $T$ that contains $x$. Let $T^{\prime}$ be the closure of this subset in $T$. We have $T^{\prime} \subset F^{\alpha_{i}}(P)$ if we choose $\varepsilon$ sufficiently small. Thus the boundary of $T^{\prime}$ is by construction contained in closure $\left(F^{\alpha_{i}}(P)-T^{\prime}\right)$ but it is also contained in $B^{\alpha_{i}-\varepsilon}(P)$. That is, we can glue $T^{\prime}$ along its boundary into both spaces. Using once more an explicit deformation retraction as described in [14] one can establish the homotopy equivalence

$$
B^{\alpha_{i}+\varepsilon}(P) \simeq B^{\alpha_{i}-\varepsilon}(P) \cup_{\varphi} T^{\prime},
$$

where $\varphi$ maps the boundary of $T^{\prime}$ onto its copy contained in $B^{\alpha_{i}-\varepsilon}(P)$. Again we are done if we show that

$$
F^{\alpha_{i}}(P)-T^{\prime} \simeq F^{\alpha_{i-1}}(P),
$$

This can be done by generalizing the technique that we used for the case that $x$ is an index 2 saddle point. We leave this generalization for the full version of this paper.

\section{CONCLUSION}

The result in this paper establishes a topological similarity between two apparently different shape constructors from a set of points. In Figure 11 we demonstrate that these shape constructors can be geometrically quite different even though they are topologically similar. The pictures for Figure 11 were produced with our implementations of efficient algorithms to compute the $\alpha$-shape and the flow shape, respectively. Also this figure shows the union of balls at the corresponding levels. Notice that this union looks almost like a big ball at large levels. The level here is so large that we had to zoom out in order to fit the union of balls on the screen.

For biological applications, namely macromolecule structure exploration, topological multi scale modeling on weighted points via $\alpha$-shapes seems to be an interesting tool [9]. The weighted points represent the positions of the atoms in the macromolecule weighted with their radii. The flow shape hierarchy can also be defined and computed efficiently for weighted points and the homotopy equivalence still holds for the weighted hierarchies. The exposition in the weighted case gets more complicated without adding anything substantial new. Thus we have decided to restrict ourselves here only to the unweighted case.

Another generalization would be to establish the results also in higher dimensions than three. This seems to be possible, but since the combinatorial complexity of both $\alpha$-shapes and flow shapes grows exponentially with the dimension, these hierarchies seem not to be interesting for applications in high dimensional space. But the case for four-dimensional space may turn out to be useful in future.

\section{REFERENCES}

[1] N. Amenta and M. Bern. Surface reconstruction by Voronoi filtering. Discr. Comput. Geom., 22 (1999), 481-504.

[2] N. Amenta, M. Bern and D. Eppstein. The crust and the $\beta$-skeleton: combinatorial curve reconstruction. Graphical Models and Image Processing 60 (1998), 125-135.

[3] N. Amenta, S. Choi, T.K. Dey and N. Leekha. A simple algorithm for homeomorphic surface reconstruction. Internat. J. Comput. Geom. \& Applications bf 12 (2002), 125-141.

[4] T.K. Dey and J. Giesen. Detecting undersampling in surface reconstruction. In Proc. 17th. ACM Sympos. Comput. Geom., (2001) 257-263.

[5] H. Edelsbrunner. The union of balls and its dual shape. Discr. Comput. Geom., 13 (1995), 415-440.

[6] H. Edelsbrunner. Surface reconstruction by wrapping finite point sets in space. Ricky Pollack and Eli Goodman Festschrift, ed. B. Aronov, S. Basu, J. Pach and M. Sharir, Springer-Verlag, to appear.

[7] H. Edelsbrunner, M. A. Facello and J. Liang. On the definition and the construction of pockets in macromolecules. Discrete Apl. Math. 88 (1998), 83-102.

[8] H. Edelsbrunner, D. G. Kirkpatrick and R. Seidel. On the shape of a set of points in the plane. IEEE Trans. 
Information Theory 29 (1983), 551-559.

[9] H. Edelsbrunner, D. Letscher and A. Zomorodian. Topological persistence and simplification. In Proc. IEEE Sympos. Found. Comput. Sci. 2000, 454-463.

[10] H. Edelsbrunner and E. P. Mücke. Three-dimensional alpha shapes. ACM Trans. Graphics 13 (1994), 43-72.

[11] J. Giesen and M. John. The Flow Complex: A Data Structure for Geometric Modeling Proc. 14th Annual ACM-SIAM Symposium on Discrete Algorithms (SODA) (2003), 285-294.
[12] K. Grove. Critical Point Theory for Distance Functions. In Proceedings of Symposia in Pure Mathematics 54(3) (1993), 357-385.

[13] J.R. Munkres. Elements of Algebraic Topology. Perseus Book Publishing, L.L.C. (1984).

[14] D. Siersma. Voronoi Diagrams and Morse Theory of the Distance Function. In Geometry in Present Day Science, O.E. Barndorff-Nielsen and E.B.V. Jensen (eds.), World Scientific (1999), 187-208. 


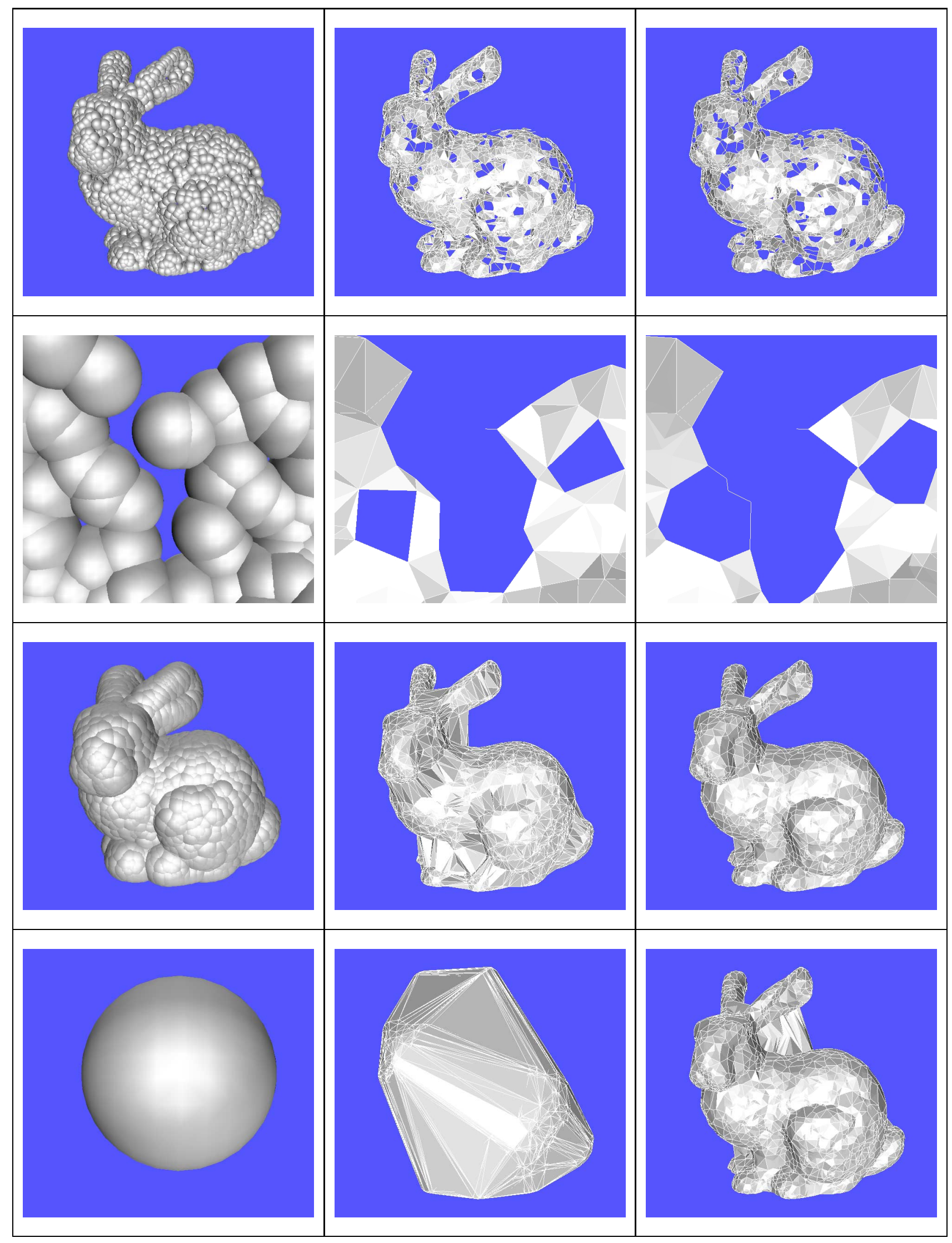

Figure 11: The union of balls (left), the $\alpha$-shape (middle) and the flow shape (right) for increasing values of $\alpha$ (top to bottom). The second row shows a zoom of the pictures in the first row. Note that the shapes in each row are homotopy equivalent. 